\title{
Fast imaging spectroscopy with MSDP spectrometers. Vector magnetic maps with THEMIS/MSDP ${ }^{\star}$
}

\author{
P. Mein ${ }^{1}$, N. Mein ${ }^{1}$, and V. Bommier ${ }^{2}$ \\ 1 Laboratoire d'Études Spatiales et d'Instrumentation en Astrophysique (LESIA), Observatoire de Paris, CNRS, UPMC, \\ Université Paris Diderot, 5 Place Jules Janssen, 92195 Meudon, France \\ e-mail: Pierre.Mein@obspm.fr \\ 2 Laboratoire d'Étude du Rayonnement et de la Matière en Astrophysique (LERMA), Observatoire de Paris, CNRS UMR 8112, \\ 5 Place Jules Janssen, 92195 Meudon, France
}

Received 8 November 2008 / Accepted 16 July 2009

\begin{abstract}
Context. Multichannel subtractive double pass (MSDP) spectrometers produce 3D data cubes $(x, y, \lambda)$ simultaneously across several line profiles. They do not suffer from image convolution by any slit width, and synchronous observations across all wavelengths avoid differential seeing effects. They are very suitable for fast 2D spectroscopy.

Aims. (1) We review specifications and capabilities of some existing MSDP spectrometers with respect to high-cadence observations. (2) THEMIS/MSDP is designed for the spectropolarimetry of strong lines. We propose new data reductions also suitable for the spectropolarimetry of photospheric lines.

Methods. An off-line algorithm is described as a way to increase the spectral resolution. Taking the opportunity of 3D data, spatial interpolations are used around each solar point by only assuming that intensity gradients $\partial I(x, y, \lambda) / \partial x$ are constant in the range $\left(x \pm \epsilon_{x}, \lambda \pm \epsilon_{\lambda}\right)$. The UNNOFIT inversion is used to compare vector magnetic maps deduced from THEMIS/MSDP and slitspectropolarimetry THEMIS/MTR data.

Results. Both results are in good agreement. In active regions, the rms of the MSDP noise, calculated over $1 \operatorname{arcsec}^{2}$, is less than $24 \mathrm{G}$ for the LOS magnetic field and less than $52 \mathrm{G}$ for $B_{x}$ and $32 \mathrm{G}$ for $B_{y}$. The MSDP scanning speed is 10 times the speed of slit-spectropolarimetry.

Conclusions. THEMIS/MSDP can provide vector magnetic maps with typical temporal resolutions that are less than 1 min for small fields-of-view and $10 \mathrm{~min}$ for active regions. This allows addressing a number of fast events.

In the future, MSDP instruments should efficiently complement single-slit spectroscopy and tunable filters. Their main capabilities should be the multiline aspect and the high temporal and spatial resolutions. New optical devices, such as image slicers, should substantially increase the signal-to-noise ratio.

For polarimetric measurements, various compromises are possible between speed, spatial resolution, and SNR. A-posteriori image restorations, either using wide band proxies or bursts of multi-wavelength short exposures, should help improving signal-to-noise ratio and spatial resolution.
\end{abstract}

Key words. instrumentation: spectrographs - techniques: polarimetric - Sun: magnetic fields

\section{Introduction}

Fast evolving structures are being investigated more and more in solar physics, especially in the low-density media of chromosphere and corona. But velocity fields and magnetic fields observed at high levels strongly depend on photospheric values, so spectropolarimetry of different wavelength ranges are often required. Time scales smaller than one minute characterize local events such as the triggering of waves across sunspots (Tziotziou et al. 2007) or emerging fluxes and Ellerman bombs in active regions (Pariat et al. 2007). But short time scales not only concern small-size events. Instabilitiy mechanisms must be investigated through magnetic field modifications across full areas of active regions. Flares, CMEs, and slip-running reconnections (Aulanier et al. 2006) require observing time scales typically as short as

\footnotetext{
* Based on observations made with the French-Italian telescope THEMIS operated by the CNRS and CNR on the island of Tenerife in the Spanish Observatorio del Teide of the Instituto de Astrofísica de Canarias.
}

one minute, while acceleration times of high-energy particles are generally shorter than one second. In this context, large data fluxes $(x, y, \lambda, t)$ are needed more and more.

Many advances have been made recently in the field of high-cadence observations by tunable filters (Cauzzi et al. 2008; Bello Gonzales et al. 2008; van Noort et al. 2008). In this paper, we concentrate on multi channel subtractive double pass (MSDP) spectrometers that produce synchronous wavelength data and avoid differential seeing effects. Unlike slit spectra, MSDP spectro-images do not suffer from any convolution by slit widths and are not affected by spatial sampling caused by scanning steps. The large scanning steps of MSDP are only used to join successive 2D-fields. They can reach high spatial resolution and provide large $3 \mathrm{D}$ data cubes $(x, y, \lambda)$ across several line profiles.

We review briefly the characteristics of some MSDP instruments, especially in view of high-cadence 2D spectroscopy. Then, we turn to spectropolarimetry and magnetic field observations. We present a new method of increasing the spectral 
Table 1. Rough characteristics of some MSDP instruments (see Sects. 2 and 3).

\begin{tabular}{|c|c|c|c|c|c|c|c|c|c|c|c|c|c|c|c|c|c|}
\hline Telescope & Mode & $N$ & $\begin{array}{r}W_{x} \\
,\end{array}$ & $\begin{array}{l}f_{\mathrm{t}} \\
\mathrm{m}\end{array}$ & $\begin{array}{l}f_{\mathrm{s}} \\
\mathrm{m}\end{array}$ & $\begin{array}{c}l \\
\mathrm{~mm}\end{array}$ & $\begin{array}{r}S \\
\mathrm{pm}\end{array}$ & $\begin{array}{r}\delta \lambda \\
\mathrm{pm}\end{array}$ & $n_{\lambda}$ & $\begin{array}{l}\Delta \lambda \\
\mathrm{nm}\end{array}$ & $\begin{array}{l}p \\
,\end{array}$ & $\begin{array}{r}\delta x \\
,\end{array}$ & $n_{x}$ & $\Delta x$ & $\Delta y$ & $\begin{array}{l}A \\
\operatorname{arcmin}^{2}\end{array}$ & $\begin{array}{r}\text { Cube } \\
n_{\lambda} A\end{array}$ \\
\hline Meudon ST & (1) & 9 & 72 & $45 / 2$ & 14 & 2.5 & 19 & 31 & 6 & 0.15 & 0.53 & 22.9 & 4 & 68.8 & 465 & 8.9 & 53.3 \\
\hline \multirow[t]{2}{*}{ German VTT } & (1) & 10 & 23 & 45 & 15 & 0.6 & 3 & 6 & 6 & 0.03 & 0.37 & 2.8 & 5 & 11.0 & 183 & 0.6 & 3.4 \\
\hline & (2) & 9 & 23 & 45 & 15 & 2.5 & 17 & 29 & 7 & 0.18 & 0.37 & 11.5 & 3 & 22.9 & 183 & 1.2 & 8.2 \\
\hline \multirow[t]{3}{*}{ Themis } & (1) & 16 & 9 & 60 & 7.7 & 0.4 & 4 & 8 & 10 & 0.08 & 0.20 & 1.4 & 7 & 8.3 & 190 & 0.44 & 4.4 \\
\hline & (2) & 9 & 9 & 60 & 7.7 & 1.2 & 9 & 27 & 7 & 0.16 & 0.20 & 4.1 & 3 & 8.3 & 190 & 0.44 & 3.0 \\
\hline & (3) & 9 & 25 & 60 & 7.7 & 1.2 & 9 & 27 & 5 & 0.11 & 0.20 & 4.1 & 5 & 16.5 & 190 & 0.87 & 4.4 \\
\hline
\end{tabular}

resolution off-line. An example of a vector magnetic map observed with THEMIS/MSDP is given and compared to results derived from single-slit spectropolarimetry.

\section{Synchronous field-of-view and data cube}

MSDP instruments are presently attached to five telescopes: Meudon ST (Solar Tower, Mein 1977), LJR (Lunette Jean Rösch) at the Pic du Midi Observatory (Mein 1981), the German VTT at Tenerife (Mein 1991), the large Wroclaw coronagraph (Rompolt et al. 1994), and THEMIS (Mein 2002). All these instruments use a double pass of the light on the same grating, except THEMIS which includes two sequential echelle spectrographs, resulting in a very low stray light level.

We restrict this short review to instruments especially suited to observing large areas with high cadences. In particular, we do not present the LJR instrument, devoted to high spatial resolution. It is built to take advantage of the high resolution of the Picdu-Midi seeing, together with the high quality of MSDP images that are not degraded by slit widths (see for example Malherbe et al. 2004; Roudier et al. 2006).

Table 1 presents some specifications in the cases of Meudon ST, German VTT, and THEMIS. For VTT and THEMIS, two lines can be observed simultaneously, and several observing modes are detailed. Modes (1) and (2) of the German VTT, and modes (1), (2), and (3) of Themis can be combined for different line strengths, as indicated in Sect. 3. $N$ and $W_{x}$ are respectively the number and the width of channels, $f_{\mathrm{t}}$ and $f_{\mathrm{s}}$ the focal lengths of telescope and spectrograph, $l$ the period of slits before the second pass, $s$ the bandwidth for each slit, $\delta \lambda$ the wavelength distance between two successive channels (that is the resulting wavelength sampling), $n_{\lambda}$ the number of channels used to cover the line profile $\Delta \lambda, p$ the pixel size (which might be reduced with CCDs larger than $1 k \times 1 k$ ), $\delta x$ the spatial shift between two successive channels, $n_{x}$ the number of channels used to cover the field-of-view $\Delta x, \Delta y$ the field-of-view in the perpendicular direction, $A$ the corresponding area and $n_{\lambda} A$ the data cube. Wavelength intervals $s, \delta \lambda$, and $\Delta \lambda$ are given for lines around $650 \mathrm{~nm}$.

Figure 1 shows an example of MSDP spectro-image obtained at the Meudon Solar Tower. In Fig. 2 we plotted the location of photons in the plane $(x, \lambda)$, where $x$ is the spatial coordinate parallel to the dispersion. The $N$ channels cover the parallelogram $a b c d$ ( $a b=$ first channel, $c d=$ last channel), but $a b c d$ is not a rectangle because the wavelength increases across each channel.

Let us derive some useful relationships between parameters characterizing MSDP instruments. The field-of-view is defined by a field stop. We call $W_{x}$ and $W_{y}$ the lengths of the entrance window. In the $y$-direction, $W_{y}$ is exactly the available fieldof-view $\Delta y$. In the $x$-direction, the available field $\Delta x$ may be

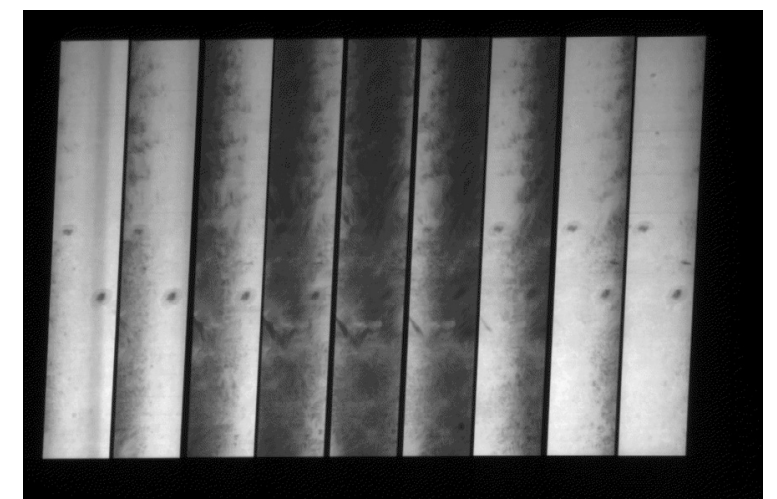

Fig. 1. Example of MSDP data in the $\mathrm{H}_{\alpha}$ line (Meudon Solar Tower, June 10, 2007, courtesy G. Molodij). The field-of-view is $465 \times$ 71 arcsec, and the wavelength distance between channels $31 \mathrm{pm}$.

smaller, according to the compromise with the total wavelength range $\Delta \lambda$ needed around line centre:

$\Delta x \leq W_{x}$.

This compromise is defined by an inscribed rectangle efgh $(e f=$ $\Delta x, e g=\Delta \lambda)$. We call $\delta l$ the physical distance between two successive slits of the "beam-shifters" that define the $N$ channels (between the first and second passes on the gratings). It corresponds to the wavelength difference $\delta \lambda$ between the photons of the same solar point in two successive channels, and also to the spatial distance $\delta x$ between two points of the Sun seen at the same wavelength in two successive channels.

For a given range of wavelengths (for example $650 \mathrm{~nm}$ as in Table 1), if the blaze angles of gratings are similar in all instruments, we can state that

$\delta \lambda=\alpha(\delta l) / f_{\mathrm{s}}$

where $f_{\mathrm{s}}$ is the equivalent focal length of the spectrograph (at the level of beam-shifters) and $\alpha$ a constant value. In a similar way

$\delta x=\beta(\delta l) / f_{\mathrm{t}}$

where $f_{\mathrm{t}}$ is the equivalent focal length of the telescope at the same level. Consequently we can write

$\delta x=(\beta / \alpha) \delta \lambda f_{\mathrm{s}} / f_{\mathrm{t}}$.

We see in Fig. 2 that

$\Delta \lambda=\left(n_{\lambda}-1\right) \delta \lambda$

and

$\Delta x=\left(n_{x}-1\right) \delta x$ 


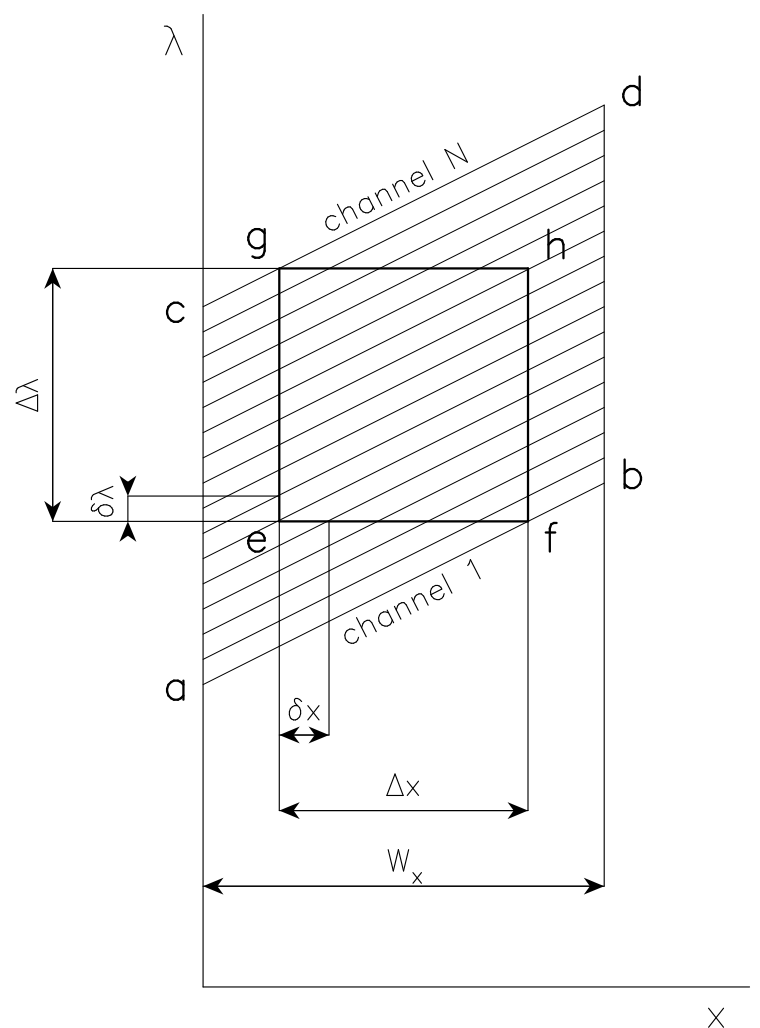

Fig. 2. Photon distribution in the $(x, \lambda)$ plane for the $N$ channels of a spectro-image (parallelogram $a b c d$ ). The rectangle $e f g h$ specifies the data covering the field of view $\Delta x$ with the wavelength range $\Delta \lambda$.

where $n_{\lambda}$ is the number of channels defining the line profile, and $n_{x}$ the number of channels necessary to cover the field-of-view for a given wavelength. We see also that

$N=n_{\lambda}+n_{x}-1$.

In the case of large solar areas, such as active regions, the full field of view is recorded by scanning, with a spatial step $\Delta x^{\prime}$ close to the width $\Delta x$, so the temporal resolution is directly connected with the total area $A \operatorname{arcmin}^{2}$ observed at a given time:

$A=\Delta x \Delta y$.

For comparison with other instruments such as tunable filters using successive exposures across a line profile, it is interesting to specify the useful Data Cube, which is is the product of $A$ by the number of synchronous wavelengths defining the useful range $\Delta \lambda$ :

Data Cube $=n_{\lambda} A$.

\section{Specific capabilities}

We again restrict this short review to the instruments listed in Table 1. The area $A$ is a clue for the speed of the instruments. From Eqs. (6) and (8) we see that

$A=\left(n_{x}-1\right) \delta x \Delta y$.

For a given $\delta \lambda$-value, the decrease in $A$ from the German VTT to THEMIS is mainly due to the decrease in $\delta x$, connected with the decrease in $f_{\mathrm{s}} / f_{\mathrm{t}}$ according to Eq. (4). The decrease from Meudon ST to the German VTT, in addition, stems from the decrease in $\Delta y$.
The MSDP of the Meudon Solar Tower is presently used for fast $\mathrm{H}_{\alpha}$ intensity and velocity maps of large active regions. The width of the line, the large focal length of the spectrograph and the focal reduction of the solar image account for a large observed field-of-view with 6 simultaneous wavelengths. A system of prisms achieves a scan of 5 successive exposures within $30 \mathrm{~s}$ and produces maps larger than $5 \times 7$ arcmin. Since 1981, many papers have been devoted to chromospheric events, such as filament oscillations (Malherbe et al. 1981), dynamical modelling of post-flare ejections (Mein \& Mein 1982) and correlations between ejecta and type-III bursts (Chiuderi-Drago et al. 1986). Recently MSDP data were used to calibrate prominence images obtained by Hinode SOT (Heinzel et al. 2008).

Two lines can be observed simultaneously with modes 1 and 2 of VTT/MSDP. Mode 1 of Table 1 is generally used for middlestrong lines (589.6 NaD1, 854.2 CaII, etc.) and mode 2 for $\mathrm{H}_{\alpha}$. Thanks to the good seeing of Tenerife, many topics were investigated, either with $\mathrm{H}_{\alpha}$ alone, such as the dynamics of arch filament systems (Mein et al. 1996) and the non-LTE inversion of prominence profiles (Molowny-Horas et al. 1999), or with $\mathrm{H}_{\alpha}$ and 854.2 CaII, such as asymmetries of flare profiles (Mein et al. 1997) and Fourier analysis of sunspot oscillations (Tziotziou et al. 2007).

Two lines can also be observed simultaneously with THEMIS/MSDP and the field-stop of $9 \times 190$ arcsec, with two possibilities. The first one uses the mode 1 for both lines: chromospheric lines (517.3 MgI, 589.6 NaD1, 587.6 HeI, 854.2 CaII, etc.) or photospheric ones (610.3 CaI, 630.2 FeI, etc. see next sections). The second possibility uses simultaneously modes 1 and 2 , the mode 2 being devoted to a Balmer line $\left(\mathrm{H}_{\alpha}\right)$. A third possibility is to use the mode 3 for $\mathrm{H}_{\alpha}$ alone, with the broad field stop $25 \times 190$ arcsec. As with VTT/MSDP, chromospheric structures were targets of published papers, as for example mottles and grains (Tziotziou et al. 2003). But, of course, since THEMIS is a polarization-free telescope, the main applications of THEMIS/MSDP concern magnetic fields. We come back to this point in subsequent sections.

\section{Observing speed: MSDP and single-slit spectroscopy}

It is interesting to qualitatively compare MSDP performances to performances of single-slit spectroscopy with respect to resolution in time. Let us concentrate on VTT (mode 1) and THEMIS (mode 1). If we assume that slit spectroscopy and MSDP use the same field-of-view versus $y$ ( $\Delta y$ value) and that the length of the target in the $y$-direction is less than $\Delta y$, a full map is obtained in both cases by a single scan versus $x$. The exposure times are generally shorter than $100 \mathrm{~ms}$, while the CDD readout and the telescope motions typically last $1 \mathrm{~s}$, so the scanning time does not depend very much on the exposure times, and the ratio of observing times is close to the ratio of the number of steps. For MSDP, scanning steps $\Delta x^{\prime}$ must be smaller than $\Delta x$ to ensure overlaps between exposures. Let us assume step sizes 10 and 5 arcsec respectively for VTT and THEMIS. If we assume that a spatial sampling around 0.5 arcsec is needed for slit spectroscopy, the corresponding scanning step is 0.5 arcsec, so we get ratios 20 and 10 for observing times between slit spectroscopy and MSDP.

Of course, slit spectroscopy provides more wavelengths than MSDP, with better spectral resolution. However, we see later that, in the case of narrow photospheric lines, methods do exist to increase the spectral resolution thanks to a little loss of spatial resolution. The resolution becomes similar to the resolution 
of typical slit spectroscopy, so that the high speed of MSDP can even be saved in that case.

\section{Circular spectropolarimetry: LOS magnetic fields}

Two MSDPs are attached to telescopes without coelostat. They are very suitable for polarimetry.

The LJR of Pic-du-Midi is a refractor. The site is wellknown for excellent seeing, and compromises are required between speed, noise, and spatial resolution to take advantage of the seeing quality. As a result, circular spectropolarimetry was achieved with LJR/MSDP by recording successive bursts of 10 couples of Stokes spectro-images $I+V$ and $I-V$. Maps of LOS magnetic field were summed after destretching (Malherbe et al. 2004). The seeing motions were deduced from local correlation tracking using images of the MSDP channel closest to the continuum (code by November 1986).

As mentioned before, we now restrict our discussion to telescopes fitted to fast scannings across large areas. THEMIS/MSDP is the other instrument allowing accurate polarimetry. The spectral resolution of mode 1 is quite suitable for measurements of Zeeman shifts of middle-strong lines. In particular, many fast maps of line-of-sight (LOS) magnetic fields were measured with THEMIS/MSDP. The scanning rate is typically 1 arcmin square per 1 min of time. Comparisons with SOHO/MDI were published (Berlicki et al. 2006). Taking advantage of the spatial resolution of MSDP spectro-images, we also analysed facular flux tubes, and published several 2D models derived from Zeeman shifts measured along the profile of $589.6 \mathrm{NaI} \mathrm{D}_{1}$ (Mein et al. 2007). LOS photospheric magnetic fields from $\mathrm{NaD}_{2}$ were also combined with $\mathrm{H}_{\alpha}$ and 854.2 CaII profiles to investigate barb endpoints of filaments (Zong et al. 2003). In a similar way, coordinated observations of $\mathrm{NaD}_{1}$ with Themis and $\mathrm{H}_{\alpha}$ with VTT have been combined to study the gradual phase of a flare (Berlicki et al. 2005).

But vector magnetic field measurements require spectral resolutions fitted to photospheric lines. The next section shows how it is possible to increase the spectral resolution of THEMIS/ MSDP off-line for that purpose.

\section{Vector magnetic fields: THEMIS/MSDP with increased spectral resolution}

The finest spectral sampling provided by THEMIS/MSDP is $8 \mathrm{pm}$ (at $600 \mathrm{~nm}$ ), with the band-width $4 \mathrm{pm}$, provided by the 16 channels optics. This is not sufficient for polarimetry of photospheric lines. Successive exposures using small grating rotations (or small shifts in the solar image) might be used to increase the number of wavelengths and to improve the sampling. Such a method is used with tunable filters. But MSDPs produce 3D data, so that it is possible to propose an alternative method that is less seeing-sensitive and that does not need any image destretching. To adapt the THEMIS/MSDP to photospheric lines, it is possible to use a compromise between spatial and spectral resolutions in the plane $(x, \lambda)$, without modifying the fast-scanning capabilities. The only condition is that the intensity gradient $\partial I(x, \lambda) / \partial x$ around a given point $x$ and a given wavelength $\lambda$ must be roughly constant in the range $x \pm \epsilon_{x}, \lambda \pm \epsilon_{\lambda}$.

Figure 3 shows the corresponding interpolations in the plane $(x, \lambda)$. The plane $(x, y)$ of 2D imagery is not represented. Wavelength scales correspond to THEMIS mode 1 of Table 1

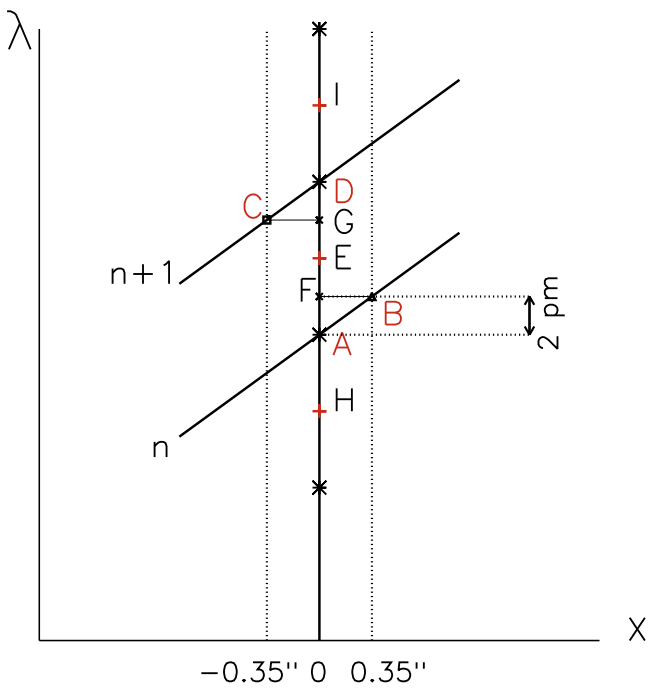

Fig. 3. Method of line-profile interpolation in the $(x, \lambda)$ plane to increase the spectral resolution of MSDP data (see Sect. 6).

with the line $610.3 \mathrm{CaI}$. For a solar point at $x=0$, line profile intensities are measured at points $\mathrm{A}$ and $\mathrm{D}$ from channels $n$ and $n+1$ (wavelengths $\lambda_{\mathrm{A}}$ and $\lambda_{\mathrm{D}}$ ). Let us call $\mathrm{B}$ and $\mathrm{C}$ the points of channels $n$ and $n+1$, at solar locations $x= \pm \epsilon_{x}$ (with $\epsilon_{x}=\delta x / 4=+0.35$ arcsec), which correspond to wavelengths $\lambda_{\mathrm{A}}+\epsilon_{\lambda}$ and $\lambda_{\mathrm{D}}-\epsilon_{\lambda}$, with $\epsilon_{\lambda}=\delta \lambda / 4 \simeq 2 \mathrm{pm}$ ). Let us call $\mathrm{F}$ and $\mathrm{G}$ the points $x=0$ at the same wavelengths as $\mathrm{B}$ and $\mathrm{C}$.

We assume that the spatial gradient of intensity $\partial I(x, y, \lambda) / \partial x$ is constant around point $\mathrm{E}\left(x=0, \lambda=\left(\lambda_{\mathrm{A}}+\lambda_{\mathrm{D}}\right) / 2\right)$, so that

$I_{\mathrm{G}}-I_{\mathrm{C}}=I_{\mathrm{B}}-I_{\mathrm{F}}$

We can replace intensities at $\mathrm{F}$ and $\mathrm{G}$ by intensities at $\mathrm{B}$ and $\mathrm{C}$ to get intensity at point $\mathrm{E}$ by a cubic interpolation. Departures due to gradients are compensated for by symmetry $(\mathrm{GE}=\mathrm{EF})$. In this way, the spectral sampling of 8 pm (distance between A and D) is reduced to $4 \mathrm{pm}$. After similar computations of intensities at $\mathrm{H}$ and $\mathrm{I}$, the final profile is obtained by new cubic interpolations between D, E, A, H for F and I, D, E, A for G.

Figure 4 shows examples of spectra deduced from MSDP data (intensities in arbitrary units, abscissae in numbers of channels).

In the case of polarimetric analysis, a grid is located at the primary focus in front of the analyser (Semel 1980). In all channels, the same fields of view are seen simultaneously in $I+S$ and $I-S(S=+Q,-Q,+U,-U,+V,-V$ in the case of beam exchange). An example of spectro-image is shown in Fig. 2 of the paper devoted to THEMIS/MSDP (Mein 2002). For each solar point, intensities are interpolated inside the CCD matrix. The pixel size is close to 0.2 arcsec, and the accuracy of the geometrical calibration is around 0.1 arcsec, so thanks to synchronous observations, interpolations for $I+S$ and $I-S$ concern the same solar areas, smoothed by the same seeing blurring, with the accuracy close to the pixel size.

\section{Full spectropolarimetry of NOAA 10960}

On June 11, 2007, the active region NOAA 10960 was observed with THEMIS/MSDP at 17:21 in the $610.27 \mathrm{~nm} \mathrm{CaI}$ line (Lande factor 2.0). The exposure time was $200 \mathrm{~ms}$ and the local bandwidth 4 pm (see Table 1). The field-of-view was $130^{\prime \prime} \times 160^{\prime \prime}$ 

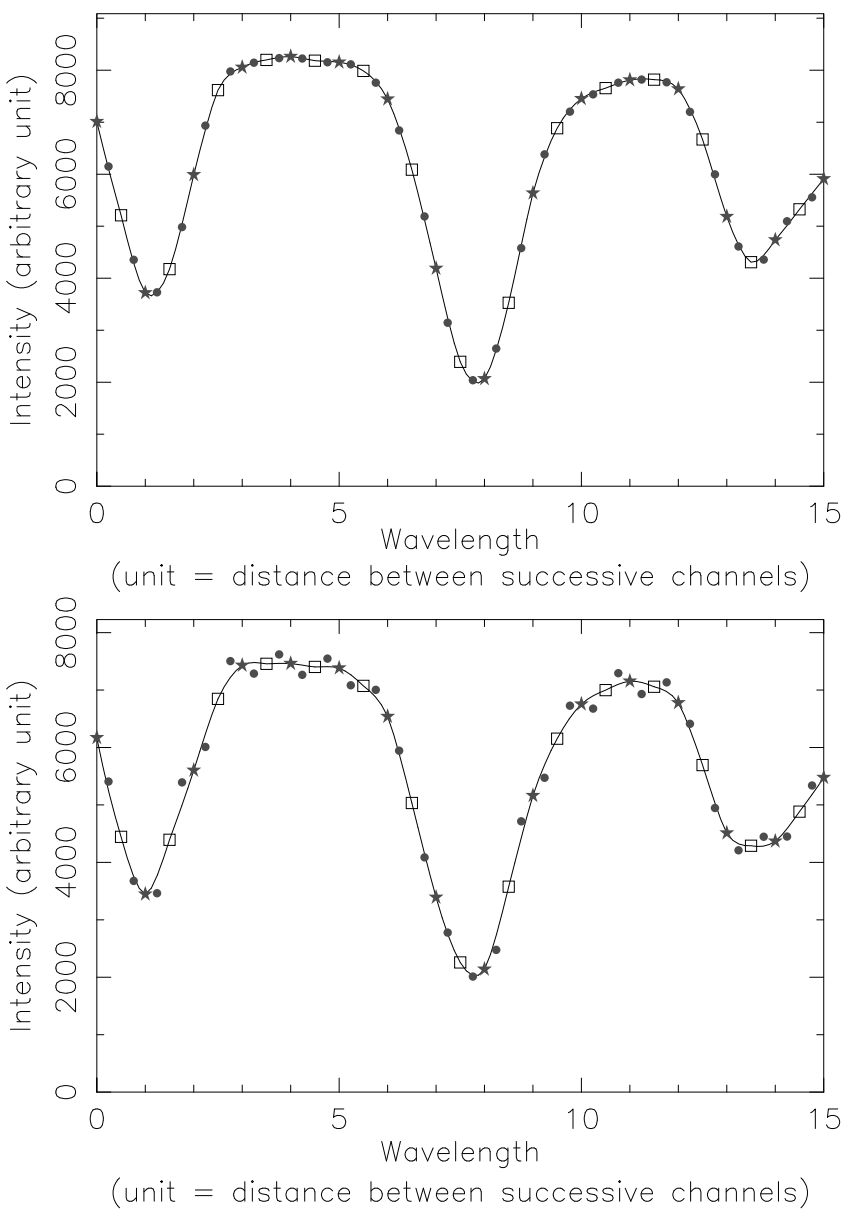

Fig. 4. Spectra derived from the 16 MSDP channels around $610.27 \mathrm{~nm}$ $\mathrm{CaI}$ (deepest line). Stars correspond to points similar to A and D in Fig. 3. Rectangles correspond to points E, H, I, and black dots to points B and C. Top: solar point with low value of $\partial(x, \lambda) / \partial x$ : black dots are on the interpolated profile. Bottom: solar point with high value of $\partial(x, \lambda) / \partial x$ : black dots are on both sides of the interpolated profile.

and the scanning time $12 \mathrm{mn}$. This corresponds to 0.5 arcmin square per minute of time. For comparison, MTR observations had been performed with the same line across a smaller field of view of the same region. The exposure time was $60 \mathrm{~ms}$ and the slit-width 1 arcsec (spectral smoothing $6 \mathrm{pm}$ ). The field-of-view was $60^{\prime \prime} \times 105^{\prime \prime}$ and the scanning time $38 \mathrm{mn}$.

The time delay between the middle points of both scans was close to $42 \mathrm{mn}$. This target was the most active solar region that we could observe in 2007. It was near the west limb (S05, W55). Between both scans, the solar rotation is about 0.4 degree. It modifies the spatial scale by less than $0.5 \%$, which corresponds to \pm 0.15 arcsec across a 60 arcsec field-of-view, and the rotation of the magnetic vector leads to errors less than 7 Gauss when the perpendicular component reaches 1000 Gauss. As a result, the solar rotation can be neglected in the comparison of MSDP and MTR results, except for the co-alignment (Sect. 9).

The polarimetry was the same in both modes. A grid was used in front of the polarization analyser, and Stokes parameters $I+S$ and $I-S$ were observed simultaneously as seen before. The grid period is 33.3 arcsec, and image restorations are obtained by 4 steps of 8.5 arcsec versus $y$. MSDP uses 5 grid periods and MTR 3 grid periods. The scanning steps versus $x$ is $5 \operatorname{arcsec}$ for MSDP and 0.7 arcsec for MTR. The $y$-scanning is performed inside the $x$-scanning loop for MSDP, but outside for MTR.

\section{Noise and photon numbers: MSDP and slit spectropolarimetries}

The CCD cameras used at THEMIS are different for MSDP and MTR, and it is difficult to directly investigate relative capabilities of the optics with respect to SNR. However, instrumental parameters can help to estimate the ratio of recorded photons in both modes, for a given solar area across the useful line profile. Let us choose for example a solar area of $1 \operatorname{arcsec}^{2}$, which is fully covered by both modes in one exposure (the slit-width of MTR is 1 arcsec). The ratio is the product of several factors, which are mainly:

- the efficiency of the interference filter that separates grating orders of MSDP (10 $\mathrm{nm}$ bandwidth), is around $70 \%$;

- four additional plane mirrors and 2 reflections in the prisms of the beam shifter (see Fig. 1 in the paper by Mein 2002) are introduced before the second pass of MSDP. Let us assume a ratio equal to 0.5 ;

- before the second pass of MSDP, the spectrum is filtered by slits of $4 \mathrm{pm}$ every $8 \mathrm{pm}$, while the full spectrum is recorded by MTR: ratio 0.5 . This ratio becomes close to 1 in the case of profile calculations with the method presented in Sect. 6, because additional data are extracted from neighbouring solar points. However, this is equivalent to a spatial smoothing and cannot be considered in the estimate of the global number of photons;

- although the first-pass grating (echelle grating, 79 grooves $/ \mathrm{mm}$, blaze 63.43 degrees) is close to the blaze angle, it is possibly less efficient than the grating used as predispersor by MTR (150 grooves/mm, blaze 2.15 degrees). We do not take it into account;

- the exposure times are $200 \mathrm{~ms}$ for MSDP and $60 \mathrm{~ms}$ for MTR: ratio 3.3.

Finally, the ratio of recorded photons in both modes during one exposure and for the same solar area is expected to be $0.70 \times$ $0.5 \times 0.5 \times 3.3 \simeq 0.6$, which corresponds to a photon noise 1.3 times greater for MSDP than for MTR. If we want to estimate the ratio of the total number of useful photons, we must take the relative area of input apertures $\Delta x \Delta y$ into account. The corresponding ratio for MSDP/MTR is $0.6 \times(8.3 / 1) \times(160 / 105)=7.6$.

\section{MSDP and MTR results with UNNOFIT inversion: departures and noise}

MSDP data were reduced with the method described in Sect. 6. In both cases, line profile inversions were performed thanks to the UNNOFIT code (Bommier et al. 2007).

The MTR data were obtained with a slit width of 1 arcsec. To get comparable results, we used a $0.5 \times 1 \operatorname{arcsec}^{2}$ smoothing of MSDP data before UNNOFIT inversion and an additional smoothing of 1 arcsec for MTR in the direction of the slit before UNNOFIT inversion. Because the interpolation method of MSDP profiles (Sect. 6) already includes additional data extracted from neighbouring solar points, we only smoothed the Stokes profiles over $0.5 \times 1 \operatorname{arcsec}^{2}$ to roughly mimic a global $1 \times 1 \operatorname{arcsec}^{2}$ smoothing.

Figures 5 and 6 show the maps of the vector magnetic field (Gauss) multiplied by the filling factor, derived from MSDP and MTR data, respectively. North is to the top, and west to the right. Scatter plots are presented in Figs. 7-9 for line-of-sight magnetic fields $B_{/ /}$and transverse components $B_{x}$ and $B_{y}$. Because the $180^{\circ}$ ambiguity is not solved, one of the transverse components 


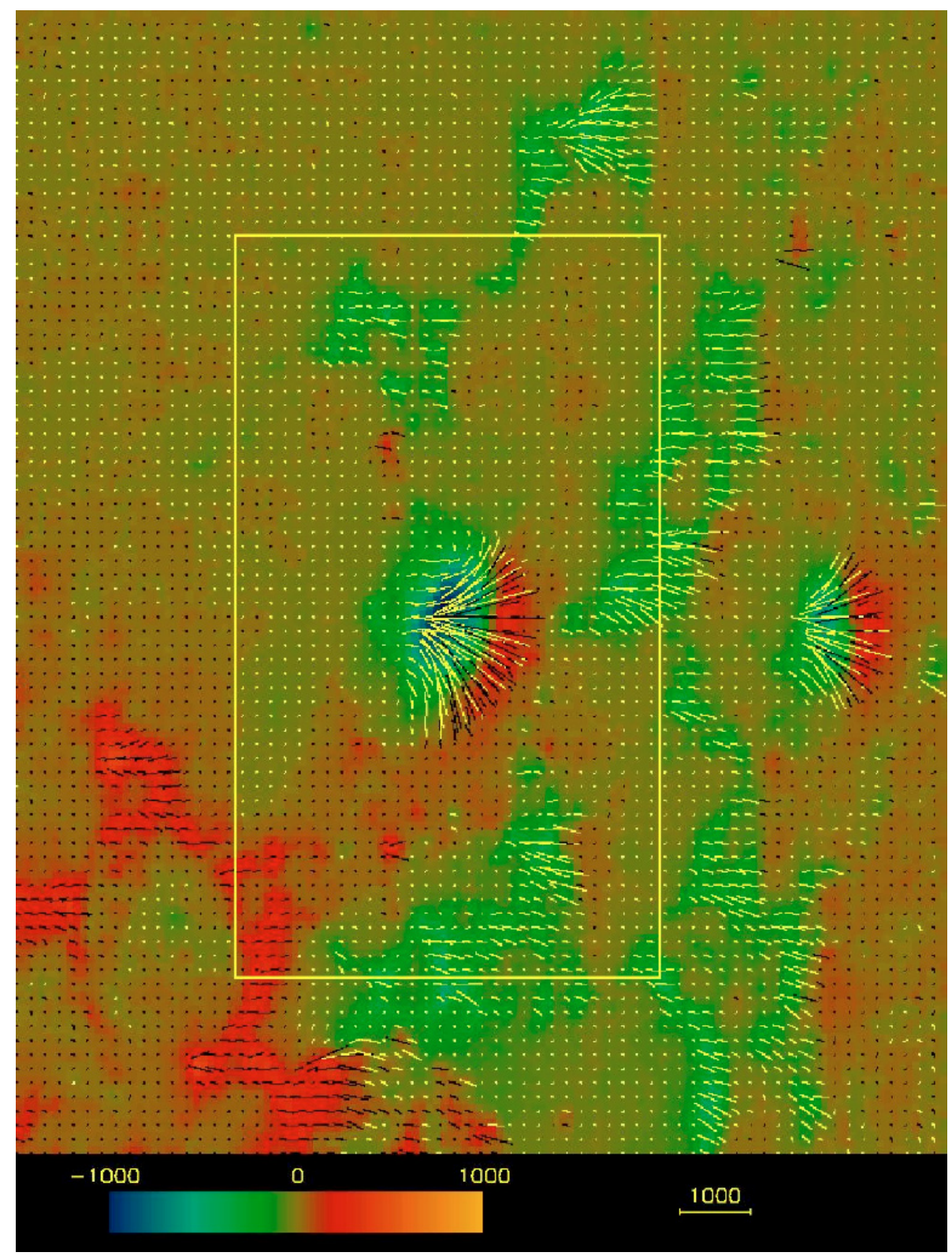

Fig. 5. Vector magnetic map derived from THEMIS/MSDP data and UNNOFIT inversion. The background colour shows the LOS magnetic field. The transverse field is represented by dashes, the length of which are proportional to the amplitude of the field (scale in Gauss). The total field-of-view is $130 \times 160$ arcsec. The rectangle of $60 \times 105$ arcsec specifies the field-of-view of MTR data.

(here the $x$-component) is always positive. Diamonds show the mean values in intervals of 100 Gauss. Vertical error bars correspond to the root mean squares of departures in each interval (counted parallel to the bisector). In most of the cases, scatter plots are well aligned along the bisector and show that mean MSDP results are close to mean MTR ones.

To compare the results more specifically, we compute, for each component, the rms of departures between MSDP and MTR values:

$d=\left\langle\left[B_{\mathrm{MTR}}(x, y)-B_{\mathrm{MSDP}}(x, y)\right]^{2}\right\rangle^{1 / 2}$

where $B$ is replaced by $B_{/ /}, B_{x}$, and $B_{y}$ successively. To distinguish active areas from the total field-of-view, the calculation is performed in two cases: $d_{0}$ is the rms averaged over the full common field-of-view and $d_{300}$ the rms averaged only over the areas where the mean modulus of the total magnetic field $|\boldsymbol{B}|$, measured in both modes, is larger than 300 Gauss. In this case, only $9 \%$ of the pixels are concerned. They correspond roughly to the spot area. Both maps, from MSDP and MTR data, have been co-aligned so as to minimize $d_{300}$ computed for $B_{/ /}$with an accuracy of \pm 0.5 arcsec.

To get an upper limit $\sigma$ of the noise, we also compute, for the three components and in the same cases, the rms of differences between the values obtained at a given point $(x, y)$ and the barycentre of values obtained at 4 neighbouring points. We define

$S=B(x+\delta x, y)+B(x-\delta x, y)+B(x, y+\delta y)+B(x, y-\delta y)$

and

$\sigma=(4 / 5)^{1 / 2}\left\langle[B(x, y)-S / 4]^{2}\right\rangle^{1 / 2}$

where $B$ is replaced successively by $B_{/ /}, B_{x}$ and $B_{y}$, for MSDP and MTR results, with $\delta x=\delta y=1$ arcsec. As before, $\sigma_{0}$ is the 


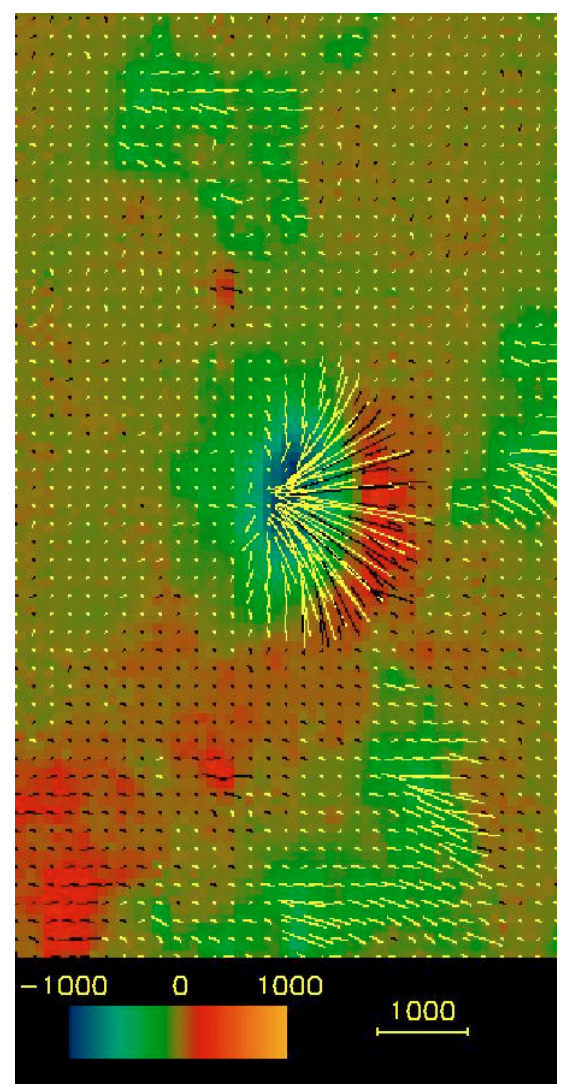

Fig. 6. Vector magnetic map derived from THEMIS/MTR data and UNNOFIT inversion. The background colour shows the LOS magnetic field. The transverse field is represented by dashes, the length of which are proportional to the amplitude of the field (scale in Gauss). The total field-of-view is $60 \times 105$ arcsec.

Table 2. Upper limits $\sigma$ of the noise and rms of departures $d$ between MSDP and MTR results, averaged over the areas where the mean value of $|B|$ is higher than 300 Gauss.

\begin{tabular}{ll|rrr}
\hline \hline & & $B_{/ /}$ & $B_{x}$ & $B_{y}$ \\
\hline$\sigma_{300}$ & MSDP & 24 & 52 & 32 \\
& MTR & 28 & 63 & 59 \\
\hline$d_{300}$ & & 94 & 190 & 145 \\
\hline
\end{tabular}

rms averaged over the full field-of-view and $\sigma_{300}$ the rms averaged only over the areas where the mean value of $|\boldsymbol{B}|$ is higher than 300 Gauss.

The $\sigma$ and $d$ values are lower for the full field than for the spot. Let us concentrate on $\sigma_{300}$ and $d_{300}$, which correspond to active areas. The $\sigma_{300}$ values are upper limits of the noise, because they also include some contribution from solar fluctuations across $x$ and $y$. For MSDP results, we get a noise less than $24 \mathrm{G}$ for $B_{/ /}, 52 \mathrm{G}$ for $B_{x}$ and $32 \mathrm{G}$ for $B_{y}$ (Table 2). For MTR, we get 28,63 , and $59 \mathrm{G}$, respectively. A large part of the MTR $\sigma$-values is due to image distortions produced by seeing and pointing effects between successive spectra (step 0.7 arcsec).

Values of $d_{300}$ are higher than $\sigma_{300}$ values: $94 \mathrm{G}$ for $B_{/ /}$, $190 \mathrm{G}$ for $B_{x}$ and $145 \mathrm{G}$ for $B_{y}$. They show some systematic departures between MSDP and MTR, probably due to the time delay between both maps and to the different scanning modes versus $x$ and $y$ (see Sect. 7) resulting in additional image distortions. Residual errors of co-alignment, slight rotations of telescope pointing, and differential seeing effects are also disturbing

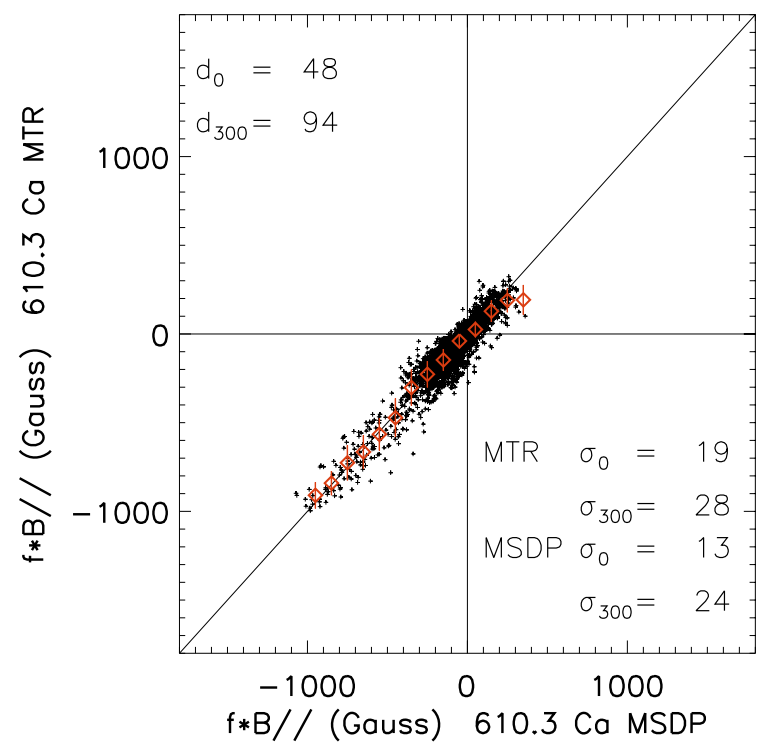

Fig. 7. Scatter plot of the LOS magnetic field multiplied by the filling factor, deduced from UNNOFIT inversion; slit-spectroscopy versus MSDP. $d_{0}$ and $d_{300}$ are the rms of departures averaged respectively over the total field-of-view and over the areas where the vector magnetic field is larger than 300 Gauss. $\sigma_{0}$ and $\sigma_{300}$ are upper limits of the noise over the same areas (see Sect. 9).

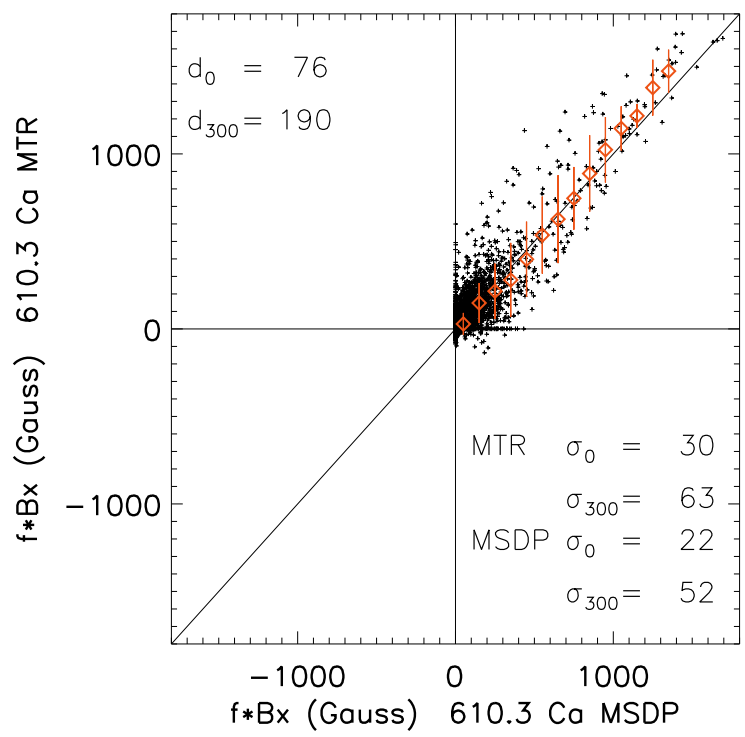

Fig. 8. Scatter plot of the component $B_{x}$ of the transverse magnetic field multiplied by the filling factor, deduced from UNNOFIT inversion; slitspectroscopy versus MSDP. Definitions as in Fig. 7.

the results. Finally, especially in the case of spots, problems may arise from the MSDP CCD camera, which is not perfectly linear at low light levels. In the future, this camera should be calibrated more accurately.

\section{Observing speeds for MSDP spectropolarimetry}

Table 3 lists some cadences of THEMIS/MSDP observations in mode 1 of Table 1 . Values of $N_{\mathrm{G}}$ and $N_{\lambda}$ are respectively the numbers of positions used to restore the full field-of-view ( $y$-steps smaller than the grid steps) and to record the Stokes parameters, $t_{\text {step }}$ and $T_{\text {tot }}$ are the scanning times for $1 x$-step and 
Table 3. Rough scanning times and temporal resolutions of THEMIS/MSDP (mode 1) for different polarimetric analysis.

\begin{tabular}{l|ll|ll|ll|l}
\hline \hline & Stokes parameters & $\begin{array}{l}I+S \text { and } I-S \\
\text { synchronous } \\
\text { (grid) }\end{array}$ & $N_{\mathrm{G}}$ & $N_{\mathrm{S}}$ & $\begin{array}{l}t_{\text {step }} \\
5^{\prime \prime} \times 160^{\prime \prime}\end{array}$ & $\begin{array}{l}T_{\text {tot }} \\
130^{\prime \prime} \times 160^{\prime \prime}\end{array}$ & $\begin{array}{l}\partial A / \partial t \\
\operatorname{arcmin}^{2} / \mathrm{mn}\end{array}$ \\
\hline 1 & $I(+Q-Q+U-U+V-V)$ & yes & 4 & 6 & $28 \mathrm{~s}$ & $12 \mathrm{mn}$ & 0.5 \\
2 & $I(+V-V)$ & yes & 4 & 2 & $14 \mathrm{~s}$ & $6 \mathrm{mn}$ & 1 \\
3 & $I(+Q-Q+U-U+V-V)$ & no & 1 & 6 & $7 \mathrm{~s}$ & $3 \mathrm{mn}$ & 2 \\
4 & $I(+V-V)$ & no & 1 & 2 & $3.5 \mathrm{~s}$ & $1.5 \mathrm{mn}$ & 4 \\
\hline
\end{tabular}

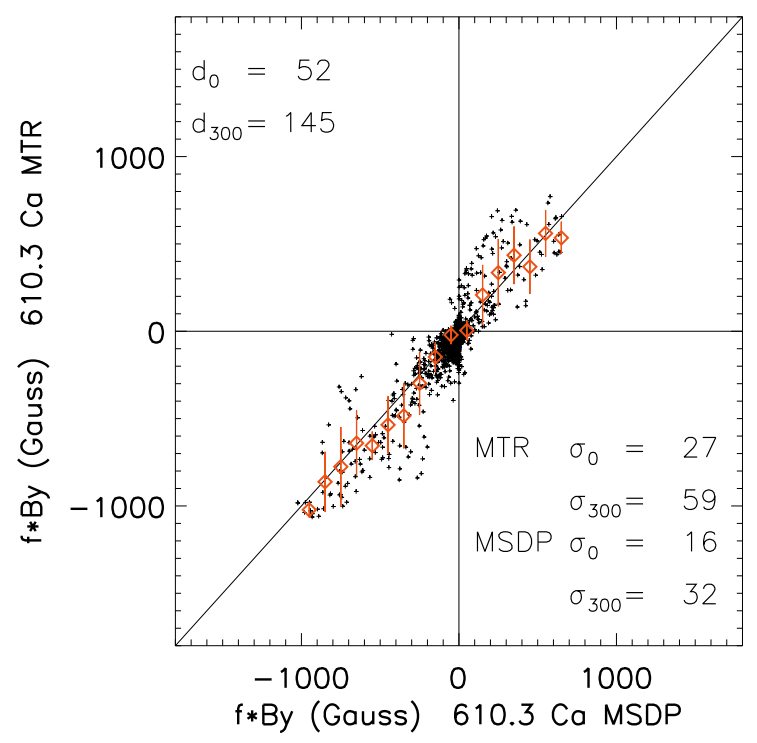

Fig. 9. Scatter plot of the component $B_{y}$ of the transverse magnetic field multiplied by the filling factor, deduced from UNNOFIT inversion; slitspectroscopy versus MSDP. Definitions as in Fig. 7.

for the total target, and $\partial A / \partial t$ is the solar area observed during 1 min of time.

Lines 1 and 2 correspond to polarimetric analysis with synchronous $I+S$ and $I-S$ (grid before the analyser). In principle $N_{\mathrm{G}}=3$ is sufficient. By taking $N_{\mathrm{G}}=4$, we get a large overlap between different parts of the field and avoid edge effects. With polarization analysis, 30 arcsec are lost at both ends of the field $\Delta y$ to determine the accurate position of field-stop edges. The effective field becomes $\Delta y^{\prime}=160^{\prime \prime}$. When $N_{\mathrm{S}}$ Stokes parameters are observed, $N_{\mathrm{G}} \times N_{\mathrm{S}}$ exposures are necessary for each scanning-step versus $x$. To save overlaps, this step is also less than $\Delta x$. It is currently $\Delta x^{\prime}=5^{\prime \prime}$ and the corresponding observed field-of-view $\Delta x^{\prime} \times \Delta y^{\prime}$ is restricted to $5^{\prime \prime} \times 160^{\prime \prime}$ for one $x$ scanning-step. Lines 3 and 4 are extrapolations to the case of successive exposures $I+S$ and $I-S$, without grid analyser and without $Y$-scanning. In lines 3 and 4 , the times are divided by $N_{\mathrm{G}}$, the number of successive exposures that were necessary to restore the field-of-view split by the grid in lines 1 and 2. But then image destretching becomes necessary before line profile inversion. Such a mode is similar to a polarization analysis with modulator, and was used at the LJR of Pic-du-Midi (Malherbe et al. 2004).

The speed of the instrument can be characterized by $\partial A / \partial t$. The full spectropolarimetry of a $5 \operatorname{arcmin}^{2}$ solar area does not need more than 10 min of time. Moreover, the spatial overlaps versus $x$ and $y$ might be reduced. In the case $N_{\mathrm{G}}=3$ and $\Delta x^{\prime}=$ 7 , the observing speed should be still increased by a factor 1.8 for items (1) and (2).

\section{Conclusion: capabilities of THEMIS/MSDP}

We detailed in Sects. 1 to 4 some basic relationships between parameters of existing MSDPs, in the context of high observing cadences. In Sects. 5 to 10, we presented fast magnetic field measurements performed with THEMIS/MSDP in mode 1 of Table 1.

The spectral resolution is suited to strong lines, and LOS magnetic fields can be measured simultaneously at 3 or 4 levels of the atmosphere, for example with lines $\mathrm{NaD} 1$ and $\mathrm{H}_{\alpha}$ (Sect. 5). But photospheric lines are required for vector magnetic fields. Section 6 details a reduction software which increases the spectral resolution by assuming some local conditions on intensity gradients, inside neighbourhoods which do not exceed $\pm 0.35^{\prime \prime}$.

To check the validity of results, observations with THEMIS/ MSDP and slit-spectroscopy THEMIS/MTR are compared in Sects. 7 to 9 . Section 10 summarizes temporal resolutions of different observing programmes of THEMIS/MSDP with mode 1 of Table 1. Performances might be improved with smaller values of $N_{\mathrm{G}}$ or faster cameras. A significant increase of cadences should be obtained also by using polarimetry modulation and image destretching. But in that case, accurate measurements imply high quality seeing.

THEMIS/MSDP can provide typical temporal resolutions smaller than $1 \mathrm{~min}$ for small fields, and $10 \mathrm{~min}$ for active regions. This allows to address a number of fast events mentioned in Sect. 1.

\section{MDSP prospects}

\subsection{Spectral resolution}

The spectral resolution is suited specifically to each one of the lines observed simultaneously. But in the case of THEMIS, it is presently limited by the slits of beam-shifters before the second pass on the gratings. It can be increased by longer focal length spectrometers, or by new optical systems. More powerful beamshifters, with better $\delta \lambda$ samplings and larger number $N$ of channels, would result also in a larger spectral coverage as well as a larger field of view. Let us note that two additional methods can be used to increase the spectral resolution. The first one is described in Sect. 6. The second one would consist in reducing the spectral sampling $\delta \lambda$ thanks to successive exposures. Small translations of beam-shifters (generally more accurate than grating rotations), can shift the wavelengths. For example, positions $-\delta x / 3,0, \delta x / 3$ would correspond to the sampling $\delta \lambda / 3$. This method is similar to the scanning used with tunable filters, but needs image restoration.

\subsection{Spatial resolution}

Mirrors of spectrographs are used over large areas in case of multi-line MSDP, and astigmatism corrections are often 
necessary to provide spatial resolutions close to the diffraction limit, as far as the spectrograph optics is concerned. Cylindrical lenses can easily be put and adjusted in front of each transfer optics of CCD cameras. Such lenses are not present in THEMIS, but only in the MSDP of VTT, with two different corrections for the two lines.

The spatial resolution of THEMIS is also limited because the telescope is not equipped with adaptive optics. Instead, a tilting mirror corrects global shifts of the solar image at relatively high frequency. It is efficient, but not well suited to observations with grid. Since the mirror is put after the first focus, the image of the grid which is located before the analyser is moving at the output of the spectrometer, and flat fields cannot be used accurately. Adaptive optics, and in particular MCAO, should take the best advantage of images provided by MSDP. But even with AO, high spatial resolution implies short exposure times, reducing the signal-to-noise ratio.

Fortunately, it is possible to combine successive 2D multiwavelength images with a "wide-band image", which can be deduced for example from the sum of all MSDP channels. A first reduction method, already used for circular polarimetry (Malherbe et al. 2004), consists in destretching by Local Correlation Tracking. Other more sophisticated ones, often used for filtergrams and based on speckle reconstructions (Keller et al. 1992; Bello Gonzalez et al. 2008; van Noort et al. 2008), can restore multi-wavelength intensity images across the line profile, before inversion. Let us note again that, while filtergrams of different wavelengths are generally obtained at different times, MSDP images of different wavelengths are simultaneous and not sensitive to differential seeing effects. Of course, fast CCD cameras are required to perform such observations.

\subsection{Temporal resolution}

The temporal resolution is proportional to the area observed synchronously, noted $A$ in Eq. (8) of Sect. 2. It can be very high, thanks to the 3D character of MSDP data, although, as we have seen before, for high spatial resolution programmes, a number of successive exposures for image restoration leads to reducing the observing speed.

It is interesting to investigate the instrumental solutions that can provide high-speed performances. We assume the best case, with the width of channels $W_{x}$ equal to the useful field $\Delta x$ (Eq. (1)). The total area of the output focal plane needed to record the $N$ channels is the sum $S$ defined by

$S=N W_{x} W_{y}=N \Delta x \Delta y=N A$.

It is determined by the size of camera mirrors and the number of lines observed simultaneously. From Eqs. (4), (6), (7), (8) we deduce

$S=\left(n_{\lambda}+\frac{\alpha}{\beta} \frac{f_{\mathrm{t}}}{f_{\mathrm{s}}} \frac{A}{\delta \lambda \Delta y}\right) A$.

For a given instrument (a given ratio $\left.\left(f_{\mathrm{t}} / f_{\mathrm{s}}\right)\right)$ and a given choice of $n_{\lambda}$ and $\delta \lambda$, we see that $A$ increases when $\Delta y$ increases. The MSDP will be faster if the field stop is more elongated $(\Delta y>$ $\Delta x)$. In the case of adaptive optics with a small circular isoplanetism area, it may be interesting to convert the entrance field of view into an elongated one. For the same $S$-value, the speed $\partial A / \partial t$ will be higher with $\Delta y=120^{\prime \prime}$ than with $\Delta y=40^{\prime \prime}$.

Furthermore, it must be noted that the present numbers of MSDP channels do not exceed 16. It is obvious that new devices should be faster with greater numbers of channels (Eqs. (6), (7), (8)).

\subsection{Signal-to-noise ratio}

Photon fluxes and SNR can be increased in different ways. According to the items of Sect. 8, we see that the number of plane mirrors between both passes should be minimized as much as possible.

But a substantial improvement would consist in reducing dark intervals between slits defining MSDP channels. This should be possible, for example, by using image slicers, which should provide, in addition, a higher spectral resolution and more channels.

\subsection{Conclusion}

New MSDPs should provide multiline observations with spectral resolution and coverage suited to each line, high spatial resolution thanks to adaptive optics and image restoration, and high temporal resolution due to $3 \mathrm{D}$ data cubes. They should efficiently complement single-slit spectroscopy and tunable filters by addressing fast-evolving solar structures with very short time scales.

Acknowledgements. We would like to thank the THEMIS team operating the telescope at Tenerife, and C. Coutard, R. Hellier, and A. Miguel, who performed many adjustments of MSDP instruments. We also thank the referee who proposed many improvements to this paper.

\section{References}

Aulanier, G., Pariat, E., Démoulin, P., \& Devore, C. R. 2006, Sol. Phys., 238, 347

Bello Gonzales, N., \& Kneer, F. 2008, A\&A, 480, 265

Berlicki, A., Schmieder, B., Vilmer, N., Aulanier, G., \& Del Zanna, G. 2004, A\&A, 423, 1119

Berlicki, A., Heinzel, P., Schmieder, B., Mein, P., \& Mein, N. 2005, A\&A, 430, 679

Berlicki, A., Mein, P., \& Schmieder, B. 2006, A\&A, 445, 1127

Bommier, V., Landi Degl'Innocenti, E., Landolfi, M., \& Molodij, G. 2007, A\&A, 464,323

Cauzzi, G., Reardon, K. P., Uitenbroek, H., et al. 2008, A\&A, 480, 515

Chiuderi-Drago, F., Mein, N., \& Pick, M. 1986, Sol. Phys., 103, 235

Heinzel, P., Schmieder, B., Farnik, F., et al. 2008, ApJ, 686, 1383

Keller, C. U., \& von der Lhe, O. 1992, A\&A, 261, 321

Malherbe, J.-M., Schmieder, B., \& Mein, P. 1981, A\&A, 102, 124

Malherbe, J.-M., Roudier, Th., Mein, P., Moity, J., \& Muller, R. 2004, A\&A, 427, 745

Mein P. 1977, Sol. Phys., 54, 45

Mein, P. 1981, Proceedings of the Japan-France seminar, ed. F. Moriyama, \& J.-C. Henoux, 285

Mein, P. 1991, A\&A, 248, 669

Mein, P. 2002, A\&A, 381, 271

Mein, P., \& Mein, N. 1982, Sol. Phys., 80, 161

Mein, P., Demoulin, P., Mein, N., et al. 1996, A\&A, 305, 343

Mein, P., Mein, N., Malherbe, J.-M., et al. 1997, Sol. Phys., 172, 161

Mein, P., Mein, N., Faurobert, M., Aulanier, G., \& Malherbe, J.-M. 2007, A\&A, 463, 727

Molowny-Horas, R., Heinzel, P., Mein, P., \& Mein, N. 1999, A\&A, 345, 618

van Noort, M., \& Rouppe van der Voort, L. 2008, A\&A, 489, 429

November, J. L. 1986, Appl. Opt., 25(3), 392

Pariat, E., Schmieder, B., Berlicki, A., \& Lopez Ariste, A. 2007, ASPC, 368, 253

Rompolt, B., Mein, P., Mein, N., Rudawy, P., \& Berlicki, A. 1994, JOSO Annual Report, 1993, 95

Roudier, Th., Malherbe, J.-M., Moity, J., et al. 2006, A\&A, 455, 1091

Semel, M. 1980, Ann. Astrophys., 91, 369

Tziotziou, K., Tsiropoula, G., \& Mein, P. 2003, A\&A, 402, 361

Tziotziou, K., Tsiropoula, G., Mein, N., \& Mein, P. 2007, A\&A, 463, 1153

Zong, W. G., Tang, Y. H., Fang, C., et al. 2003, A\&A, 412, 267 